\title{
On the Error Rate Analysis of Dual-Hop Amplify-and-Forward Relaying in Generalized-K Fading Channels
}

\author{
George P. Efthymoglou, ${ }^{1}$ Nikolaos Bissias, ${ }^{1}$ and Valentine A. Aalo ${ }^{2}$ \\ ${ }^{1}$ Department of Digital Systems, University of Piraeus, 80 Karaoli and Dimitriou Street, Piraeus 18534, Greece \\ ${ }^{2}$ Department of Computer and Electrical Engineering and Computer Science, Florida Atlantic University, Boca Raton, FL 33431, USA
}

Correspondence should be addressed to George P. Efthymoglou,gefthymo@unipi.gr

Received 30 November 2009; Accepted 5 March 2010

Academic Editor: Nikos Sagias

Copyright (c) 2010 George P. Efthymoglou et al. This is an open access article distributed under the Creative Commons Attribution License, which permits unrestricted use, distribution, and reproduction in any medium, provided the original work is properly cited.

\begin{abstract}
We present novel and easy-to-evaluate expressions for the error rate performance of cooperative dual-hop relaying with maximal ratio combining operating over independent generalized- $K$ fading channels. For this system, it is hard to obtain a closed-form expression for the moment generating function (MGF) of the end-to-end signal-to-noise ratio (SNR) at the destination, even for the case of a single dual-hop relay link. Therefore, we employ two different upper bound approximations for the output SNR, of which one is based on the minimum SNR of the two hops for each dual-hop relay link and the other is based on the geometric mean of the SNRs of the two hops. Lower bounds for the symbol and bit error rates for a variety of digital modulations can then be evaluated using the MGF-based approach. The final expressions are useful in the performance evaluation of amplify-and-forward relaying in a generalized composite radio environment.
\end{abstract}

\section{Introduction}

Cooperative diversity with relays has been shown to provide high data rate coverage and mitigate channel impairments in next generation wireless systems. Amplify-and-forward relay techniques have attracted a lot of attention recently as they provide a simple way to implement collaborative/cooperative wireless communication systems. For dual-hop nonregenerative systems, the end-to-end signal-to-noise ratio (SNR) at the receiving node depends on the amplification gain employed at the relays. For relays with channel side information (CSI) of the first link, the end-to-end SNR of a single dual-hop relay link has been obtained in [1]. For this relay transmission scenario, analytical performance results have been obtained by approximating the end-to-end SNR by the harmonic mean of the SNRs of the two hops [2], their geometric mean [3], and the minimum SNR of the two hops $[4,5]$. Among the proposed approximations for the endto-end SNR of dual-hop transmission, the harmonic mean and the minimum SNR bounds have been shown to result in tight performance bounds $[2,4]$, whereas the geometric mean bound has been shown to give accurate results for low and medium values of the SNR per hop [3,4]. Using one of the above proposed upper bounds for the total SNR, the performance of dual-hop relaying has been studied in terms of outage probability and average bit error rate (BER) for various symmetrical fading conditions, such as Rayleigh [1], Nakagami- $m$ [2-4], Weibull [5], and generalized Gamma [6] fading, as well as for asymmetrical links [7, 8], although most analyses have been restricted to single dual-hop relay links.

The generalized- $K$ fading model [9] has also attracted considerable attention as one of the most general wireless fading models that can characterize the combined effects of fast and slow fading on the received signal. This fading model corresponds to a Nakagami-Gamma composite distribution and is controlled by two shaping parameters $m$ and $k$, where $m$ is the Nakagami parameter for the short-term fading and $k$ is the parameter of the gamma distribution for the received average power due to shadowing [9]. Note that the $K$ distribution [10] is derived as a special case of the generalized- $K$ distribution by letting $m=1$ (i.e., Rayleigh short-term fading). A number of results on the performance analysis of communication links in this fading model can be found in the literature $[11,12]$. 
Recently, analytical expressions for the error rate performance of dual-hop relaying over generalized- $K$ fading channels were given in terms of convergent infinite series in [13], using the minimum SNR upper bound for the end-toend SNR and averaging the conditional BER over the derived probability density function (pdf) of the total SNR. However, these expressions are restricted to a single dual-hop relay system and result in some truncation error depending on the number of terms employed. Furthermore, the expressions in [13] cannot be evaluated for integer values of the shaping parameter $k$. In this paper, using both the minimum SNR and the geometric mean upper bounds for the end-toend SNR of a single relay link and employing the moment generating function (MGF) based approach, we present novel expressions for the error rate performance of multiple dual-hop relaying with MRC operating over independent generalized- $K$ fading channels with integer values of fading parameter $m$ and arbitrary values of fading parameter $k$. Note that using the geometric mean approximation of the total SNR, independent non-identical fading in the two hops of each relay, that is, source-to-relay and relayto-destination, can be considered, whereas the minimum SNR performance bound is restricted to independent and identically distributed (i.i.d.) fading channels.

The rest of the paper is organized as follows. In Section 2 we present the statistics of the generalized- $K$ distribution, that is, the pdf, cumulative density function (cdf), and MGF of the instantaneous received SNR of a single direct link. In Section 3, using the MGF-based approach, we derive the average symbol error rate (SER) of multiple dual-hop relay links with MRC at the receiver side. Numerical and simulation results are given in Section 4, while concluding remarks are given in Section 5.

\section{Statistics of the Generalized-K Distribution}

We assume that the fading environment is such that the signal envelope $X$ in a receive antenna is a generalized- $K$ distributed random variable with pdf given by [9]

$$
\begin{aligned}
f_{X}(x)= & \frac{4 m^{(k+m) / 2}}{\Gamma(m) \Gamma(k) \Omega^{(k+m) / 2}} x^{k+m-1} \\
& \times K_{k-m}\left(2\left(\frac{m}{\Omega}\right)^{1 / 2} x\right), \quad x \geq 0,
\end{aligned}
$$

where $k$ and $m$ are the distribution's shaping parameters, $\Omega=$ $E\left[X^{2}\right] / k$ is the mean power with $\mathrm{E}[\cdot]$ denoting expectation, $\Gamma(\cdot)$ is the Gamma function, and $K_{k-m}(\cdot)$ is the $(k-m)$ th order modified Bessel function of the second kind [14].

The instantaneous received SNR per symbol of a single receiver is $\gamma=X^{2} E_{s} / N_{0}$, where $E_{s}$ is the symbol energy and $N_{0}$ is the single-sided power spectral density of the additive white Gaussian noise (AWGN). The corresponding average received SNR per symbol is given as $\bar{\gamma}=k \Omega \cdot E_{s} / N_{0}$. The pdf of $\gamma$ is given by

$$
f_{\gamma}(\gamma)=\frac{2 \Xi^{(k+m) / 2}}{\Gamma(m) \Gamma(k)} \gamma^{(k+m) / 2-1} K_{k-m}(2 \sqrt{\Xi \gamma}), \quad \gamma \geq 0,
$$

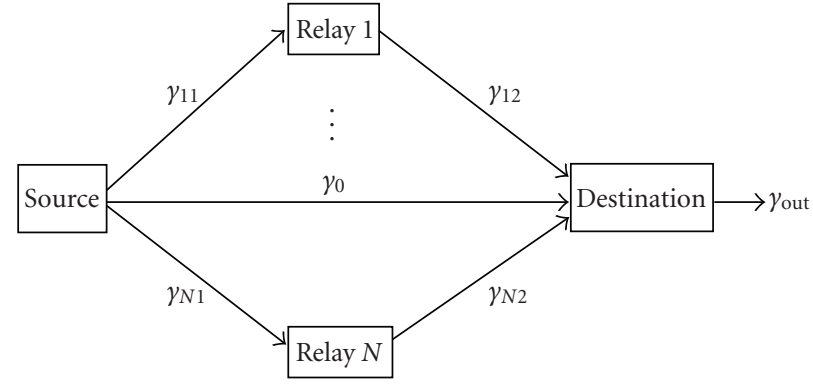

Figure 1: Cooperative dual-hop relay transmission scheme with MRC at the destination.

with $\Xi=(k m) / \bar{\gamma}$. The cdf of $\gamma$, defined as $F_{\gamma}(\gamma)=\int_{0}^{\gamma} f_{\gamma}(x) d x$, has been obtained in [15] for integer values of $m$ and arbitrary values of $k$, as

$$
F_{\gamma}(\gamma)=1-\frac{2(\Xi \gamma)^{k / 2}}{\Gamma(k)} \sum_{r=0}^{m-1} \frac{1}{r !}(\Xi \gamma)^{r / 2} K_{k-r}(2 \sqrt{\Xi \gamma}), \quad \gamma \geq 0 .
$$

Moreover, the MGF of $\gamma$, defined as $\mathcal{M}_{\gamma}(-s)=$ $\int_{0}^{\infty} e^{-s \gamma} f_{\gamma}(\gamma) d \gamma$, is given by [15]

$$
\mathcal{M}_{\gamma}(-s)=\frac{1}{\Gamma(m) \Gamma(k)} G^{2,1}\left(\frac{s}{\Xi} \mid \begin{array}{c}
1-k, 1-m \\
0
\end{array}\right),
$$

where $G(\cdot)$ is the Meijer's $G$-function [14, equation (9.301)].

\section{End-to-End Error Rate Analysis}

We consider a dual-hop relay system with $N$ relays as well as a direct link between the source and the destination, as shown in Figure 1. The output SNR, assuming MRC at the destination receiving end, can be written as

$$
\gamma_{\text {out }}=\gamma_{0}+\sum_{\ell=1}^{N} \gamma_{\text {end }}(\ell)
$$

where $\gamma_{0}$ is the SNR of the direct link and $\gamma_{\text {end }}(\ell)$ is the end-to-end SNR of the $\ell$ th relay. For amplify-andforward relays with CSI at the relays, $\gamma_{\text {end }}(\ell)$ is known to be given by the harmonic mean of the three positive random variables $\left(\gamma_{\ell 1}, \gamma_{\ell 2}, \gamma_{\ell 1} \gamma_{\ell 2}\right)$, that is, $\gamma_{\mathrm{end}}(\ell)=\gamma_{\ell 1} \gamma_{\ell 2} /\left(\gamma_{\ell 1}+\gamma_{\ell 2}+\right.$ 1) [1]. Assuming independent fading in all relay links, the MGF of the output SNR is then given by

$$
\mathcal{M}_{\gamma_{\text {out }}}(-s)=\mathcal{M}_{\gamma_{0}}(-s) \prod_{\ell=1}^{N} \mathcal{M}_{\gamma_{\text {end }}(\ell)}(-s) \text {. }
$$

For the generalized- $K$ fading environment, $\mathcal{M}_{\gamma_{0}}(-s)$ is given by (4). In order to obtain mathematically tractable results for the MGF of $\gamma_{\text {end }}(\ell)$, the end-to-end SNR for the relay channel has been approximated by different upper bounds [2-4]. In this paper, for the generalized- $K$ fading environment, we consider the approximations of minimum SNR [4] and geometric mean [3]. 
It follows that the average SER and BER performances for a variety of digital modulations can be evaluated using the MGF-based approach. For example, the average SER for $M$-ary phase-shift keying ( $M$-PSK) is given by

$$
P_{e, \mathrm{MPSK}}=\frac{1}{\pi} \int_{0}^{(M-1) \pi / M} \mathcal{M}_{\text {yout }}\left(-\frac{g_{M}}{\sin ^{2} \theta}\right) d \theta,
$$

where $g_{M}=\sin ^{2}(\pi / M)$, whereas for $M$-ary quadrature amplitude modulation ( $M$-QAM) the average SER is given by

$$
\begin{aligned}
P_{e, \text { MQAM }}= & \frac{4 q}{\pi} \int_{0}^{\pi / 2} \mathcal{M}_{\gamma_{\text {out }}}\left(-\frac{g_{Q}}{\sin ^{2} \theta}\right) d \theta \\
& -\frac{4 q^{2}}{\pi} \int_{0}^{\pi / 4} \mathcal{M}_{\gamma_{\text {out }}}\left(-\frac{g_{Q}}{\sin ^{2} \theta}\right) d \theta,
\end{aligned}
$$

where $q=1-1 / \sqrt{M}$ and $g_{Q}=3 /(2(M-1))$.

\subsection{Performance Using the Minimum SNR Approximation.} The end-to-end SNR of a dual-hop relay system with multiple relays and a direct link between the source and the destination can be approximated by its upper bound $\gamma_{a}$ as follows [4]:

$$
\gamma_{\text {out }} \leq \gamma_{a}=\gamma_{0}+\sum_{\ell=1}^{N} \gamma_{\min }(\ell)
$$

where $\gamma_{\min }(\ell)=\min \left(\gamma_{\ell 1}, \gamma_{\ell 2}\right), \ell=1, \ldots, N$. The pdf of $\gamma_{\min }(\ell)$ is given by [14]

$$
\begin{aligned}
f_{\gamma_{\min }(\ell)}(\gamma)= & f_{\gamma_{\ell 1}}(\gamma)+f_{\gamma_{e 2}}(\gamma) \\
& -\left[f_{\gamma_{\ell 1}}(\gamma) F_{\gamma_{e 2}}(\gamma)+F_{\gamma_{\ell 1}}(\gamma) f_{\gamma_{e 2}}(\gamma)\right] .
\end{aligned}
$$

Using (10), the MGF of $\gamma_{\min }(\ell)$ is given by

$$
\begin{aligned}
\mathcal{M}_{\gamma_{\text {min }}(\ell)}(-s)= & \int_{0}^{\infty} e^{-s \gamma_{\min }(\ell)} f_{\gamma_{\min }(\ell)}(\gamma) d \gamma \\
= & \mathcal{M}_{\gamma_{\ell 1}}(-s)+\mathcal{M}_{\gamma_{\ell 2}}(-s) \\
& -\left[\mathcal{M}_{\gamma_{\ell 12}}(-s)+\mathcal{M}_{\gamma_{\ell 21}}(-s)\right],
\end{aligned}
$$

where

$$
\mathcal{M}_{\gamma_{\ell i j}}(-s)=\int_{0}^{\infty} e^{-s \gamma} f_{\gamma_{\ell i}}(\gamma) F_{\gamma_{\ell j}}(\gamma) d \gamma
$$

for $i, j \in\{1,2\}$. In order to derive closed form analytical results, we consider i.i.d. fading for the source-to-relay and relay-to-destination links. Using (3) in (12) we obtain

$$
\mathcal{M}_{\gamma_{\ell i j}}(-s)=\mathcal{M}_{\gamma_{\ell i}}(-s)-I(-s) \text {, }
$$

where

$$
\begin{aligned}
I(-s)= & \frac{4 \Xi^{(2 k+m) / 2}}{\Gamma(m) \Gamma^{2}(k)} \\
& \times \sum_{r=0}^{m-1} \frac{1}{r !}(\Xi)^{r / 2} \int_{0}^{\infty} e^{-s \gamma} \gamma^{k+(m+r) / 2-1} \\
& \quad \times K_{k-m}(2 \sqrt{\Xi \gamma}) K_{k-r}(2 \sqrt{\Xi \gamma}) d \gamma .
\end{aligned}
$$

Note that in (14) we dropped the subscripts on $k, m$, and $\Xi$, for simplicity. By expressing the product of two Bessel $K$-functions in terms of the Meijer $G$-function using [16, equation (03.04.26.0016.01)] and evaluating the resulting integral using [14, equation (7.813.1)], followed by the functional relationships [14, equation (9.31.5)] and [14, equation (9.31.2)], the final result is given by

$$
\begin{aligned}
I(-s) & =\frac{\sqrt{\pi}}{2^{2 k+m-1} \Gamma(m) \Gamma^{2}(k)} \sum_{r=0}^{m-1} \frac{1}{2^{r} r !} \\
& \times G_{4,3}^{1,4}\left(\frac{s}{4 \Xi} \mid \begin{array}{l}
1-2 k, 1-k-r, 1-k-m, 1-m-r \\
0,1-k-\frac{m+r}{2},-k-\frac{m+r-1}{2}
\end{array}\right) .
\end{aligned}
$$

Therefore, assuming i.i.d. fading in the two hops of the $\ell$ th relay link, for $\ell=1, \ldots, N$, the MGF of $\gamma_{\min }(\ell)$ in (11) becomes $\mathcal{M}_{\gamma_{\min }(\ell)}(-s)=2 I(-s)$, that is,

$$
\begin{aligned}
& \mathcal{M}_{\gamma_{\min }(\ell)}(-s)=\frac{\sqrt{\pi}}{2^{2 k_{\ell}+m_{\ell}-2} \Gamma\left(m_{\ell}\right) \Gamma^{2}\left(k_{\ell}\right)} \sum_{r=0}^{m_{\ell}-1} \frac{1}{2^{r} r !} \\
& \times G_{4,3}^{1,4}\left(\begin{array}{ll}
\left.\frac{s}{4 \Xi_{\ell}} \mid \begin{array}{l}
1-2 k_{\ell}, 1-k_{\ell}-r, 1-k_{\ell}-m_{\ell}, 1-m_{\ell}-r \\
0,1-k_{\ell}-\frac{m_{\ell}+r}{2},-k_{\ell}-\frac{m_{\ell}+r-1}{2}
\end{array}\right) .
\end{array}\right.
\end{aligned}
$$

Finally, the MGF of the MRC output SNR is approximated by

$$
\mathcal{M}_{\gamma_{\alpha}}(-s)=\mathcal{M}_{\gamma_{0}}(-s) \prod_{\ell=1}^{N} \mathcal{M}_{\gamma_{\min }(\ell)}(-s) .
$$

3.2. Performance Using the Geometric Mean Approximation. It is well known that the end-to-end SNR of the $\ell$ th relay link can be expressed in terms of the harmonic mean of the three positive random variables $\left(\gamma_{\ell 1}, \gamma_{\ell 2}, \gamma_{\ell 1} \gamma_{\ell 2}\right)$. Following [3], the end-to-end SNR can be upper bounded using the geometric mean of $\left(\gamma_{\ell 1}, \gamma_{\ell 2}, \gamma_{\ell 1} \gamma_{\ell 2}\right)$, as

$$
\gamma_{\text {out }} \leq \gamma_{b}=\gamma_{0}+\frac{1}{3} \sum_{\ell=1}^{N}\left(\gamma_{\ell 1} \gamma_{\ell 2}\right)^{2 / 3} .
$$

By letting $\gamma_{\ell}=(1 / 3)\left(\gamma_{\ell 1} \gamma_{\ell 2}\right)^{2 / 3}$, the MGF of $\gamma_{\ell}$ is given by

$$
\mathcal{M}_{\gamma_{\ell}}(-s)=\int_{0}^{\infty} \int_{0}^{\infty} e^{-(s / 3)\left(\gamma_{1} \gamma_{2}\right)^{2 / 3}} f_{\gamma_{\ell 1}}\left(\gamma_{1}\right) f_{\gamma_{\ell 2}}\left(\gamma_{2}\right) d \gamma_{1} d \gamma_{2}
$$

By replacing (2) in (19) we obtain (note that in the derivations, for simplicity, we drop the subscript $\ell$ on the fading parameters)

$$
\begin{aligned}
\mathcal{M}_{\gamma_{e}}(-s)= & \frac{4 \Xi_{1}^{\left(k_{1}+m_{1}\right) / 2} \Xi_{2}^{\left(k_{2}+m_{2}\right) / 2}}{\Gamma\left(m_{1}\right) \Gamma\left(m_{2}\right) \Gamma\left(k_{1}\right) \Gamma\left(k_{2}\right)} \\
& \times \int_{0}^{\infty} \int_{0}^{\infty} e^{-(s / 3) \gamma_{1}^{2 / 3} \gamma_{2}^{2 / 3}} \gamma_{1}^{\left(k_{1}+m_{1}\right) / 2-1} \gamma_{2}^{\left(k_{2}+m_{2}\right) / 2-1} \\
& \quad \times K_{k_{1}-m_{1}}\left(2 \sqrt{\Xi_{1} \gamma_{1}}\right) K_{k_{2}-m_{2}}\left(2 \sqrt{\Xi_{2} \gamma_{2}}\right) d \gamma_{1} d \gamma_{2} .
\end{aligned}
$$


Following a similar procedure to the one in [3], the inner integral is given by

$$
I_{1}(-s)=\int_{0}^{\infty} e^{-(s / 3) \gamma_{1}^{2 / 3} \gamma_{2}^{2 / 3}} \gamma_{1}^{\left(k_{1}+m_{1}\right) / 2-1} K_{k_{1}-m_{1}}\left(2 \sqrt{\Xi_{1} \gamma_{1}}\right) d \gamma_{1}
$$

By substituting $K_{v}(2 \sqrt{x})=(1 / 2) G_{0,2}^{2,0}(x \mid v / 2,-v / 2)$ and $e^{-x}=G_{0,1}^{1,0}(x \mid \overline{0})$ in (21) and using [16, equation (07.34.21.0013.01)], we obtain the closed-form result

$$
\begin{aligned}
& I_{1}(-s)=\frac{\sqrt{3} 2^{k_{1}+m_{1}}}{2^{4} \pi^{2} \Xi_{1}^{\left(k_{1}+m_{1}\right) / 2}} \\
& \times G_{4,3}^{3,4}\left(\begin{array}{c|c}
\frac{2^{4} s^{3} \gamma_{2}^{2}}{3^{6} \Xi_{1}^{2}} & \Delta\left(2, k_{1}\right), \Delta\left(2, m_{1}\right) \\
0, \frac{1}{3}, \frac{2}{3}
\end{array}\right),
\end{aligned}
$$

where $\Delta(\cdot, \cdot)$ is defined as $\Delta(p, \mu)=\{(1-\mu) / p, \ldots,(p-$ $\mu) / p$, with $p$ being positive integer and $\mu$ positive real. Using (22), the outer integral in (20) becomes

$$
\begin{aligned}
I_{2}(-s)= & \frac{\sqrt{3} 2^{k_{1}+m_{1}}}{2^{4} \pi^{2} \Xi_{1}^{\left(k_{1}+m_{1}\right) / 2}} \\
& \times \int_{0}^{\infty} G_{4,3}^{3,4}\left(\frac{2^{4} s^{3} \gamma_{2}^{2}}{3^{6} \Xi_{1}^{2}} \mid \begin{array}{c}
\Delta\left(2, k_{1}\right), \Delta\left(2, m_{1}\right) \\
0, \frac{1}{3}, \frac{2}{3}
\end{array}\right) \\
& \quad \times \gamma_{2}^{\left(k_{2}+m_{2}\right) / 2-1} K_{k_{2}-m_{2}}\left(2 \sqrt{\Xi_{2} \gamma_{2}}\right) d \gamma_{2} .
\end{aligned}
$$

Again, expressing the Bessel $K$-function in terms of the $G$-function and using [16, equation (07.34.21.0013.01)], followed by some straightforward manipulations, we obtain

$$
\begin{aligned}
& I_{2}(-s)=\frac{\sqrt{3} 2^{k_{1}+m_{1}+k_{2}+m_{2}}}{2^{7} \pi^{3} \Xi_{1}^{\left(k_{1}+m_{1}\right) / 2} \Xi_{2}^{\left(k_{2}+m_{2}\right) / 2}} \\
& \quad \times G_{8,3}^{3,8}\left(\frac{2^{8} s^{3}}{3^{6} \Xi_{1}^{2} \Xi_{2}^{2}} \mid \begin{array}{c}
\Delta\left(2, k_{1}\right), \Delta\left(2, m_{1}\right), \Delta\left(2, k_{2}\right), \Delta\left(2, m_{2}\right) \\
0, \frac{1}{3}, \frac{2}{3}
\end{array}\right) .
\end{aligned}
$$

Using the above result for $I_{2}(-s)$, the MGF of $\gamma_{\ell}$ is given by

$$
\begin{aligned}
& \mathcal{M}_{\gamma_{\ell}}(-s)=\frac{\sqrt{3} 2^{k_{\ell 1}+m_{\ell 1}+k_{\ell 2}+m_{\ell 2}}}{2^{5} \pi^{3} \Gamma\left(m_{\ell 1}\right) \Gamma\left(m_{\ell 2}\right) \Gamma\left(k_{\ell 1}\right) \Gamma\left(k_{\ell 2}\right)} \\
& \times G_{8,3}^{3,8}\left(\frac{2^{8} s^{3}}{3^{6} \Xi_{\ell 1}^{2} \Xi_{\ell 2}^{2}} \mid \begin{array}{c}
\Delta\left(2, k_{\ell 1}\right), \Delta\left(2, m_{\ell 1}\right), \Delta\left(2, k_{\ell 2}\right), \Delta\left(2, m_{\ell 2}\right) \\
0, \frac{1}{3}, \frac{2}{3}
\end{array}\right) .
\end{aligned}
$$

Finally, owing to the independency of $\gamma_{\ell}, \ell=1, \ldots, N$, the MGF of the MRC output SNR is approximated by the product of the MGFs, as

$$
\mathcal{M}_{\gamma_{\mathrm{b}}}(-s)=\mathcal{M}_{\gamma_{0}}(-s) \prod_{\ell=1}^{N} \mathcal{M}_{\gamma_{\ell}}(-s) \text {. }
$$

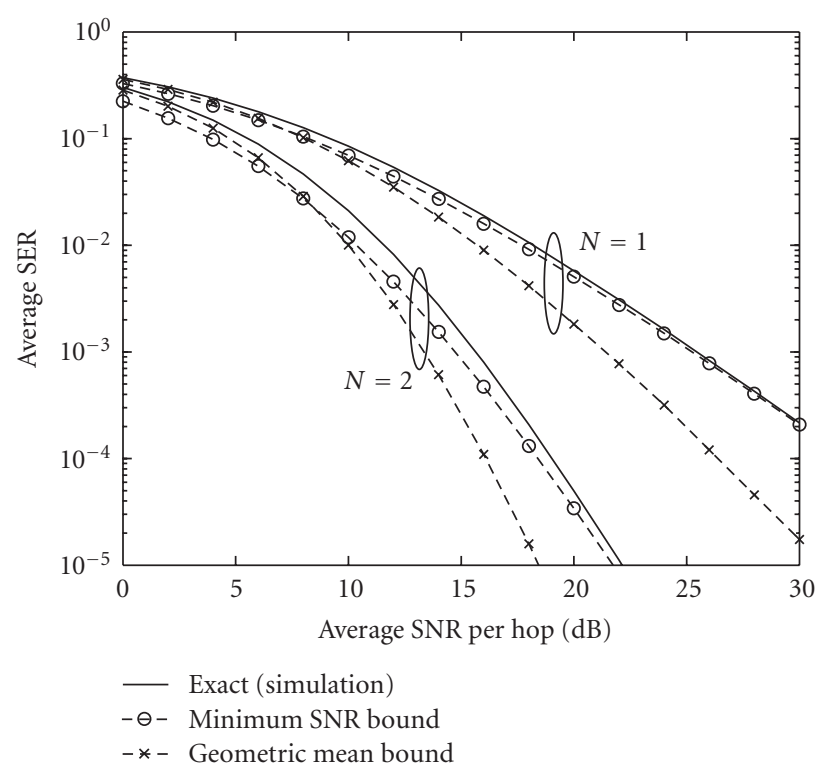

FIGURE 2: Average SER for 4-PSK versus average SNR per hop for $N=1$ and $N=2$ dual-hop links with MRC assuming $m_{0}=1, k_{0}=$ 0.5 for the direct link, $m_{1}=1, k_{1}=1.5$ for the first relay link, and $m_{2}=2, k_{2}=3$ for the second relay link.

\section{Numerical Results}

In this section we present some numerical and simulation results on the error rate performance of the cooperative dual-hop relay transmission scheme with MRC operating over independent generalized- $K$ fading channels. This fading model corresponds to a Nakagami-Gamma composite distribution and is controlled by two shaping parameters $m$ and $k$, where the parameter $m \geq 1 / 2$ inversely reflects the multipath fading severity and the positive parameter $k$ inversely reflects the shadowing severity [9]. For demonstration purposes, we assume different fading conditions for each relay link, that is, $m_{0}=1, k_{0}=0.5$ for the direct link, $m_{1}=1, k_{1}=1.5$ for the first relay link, and $m_{2}=2, k_{2}=3$ for the second relay link. Using the MGF-based approach for performance evaluation over fading channels and the two approximations for the end-to-end SNR of a single dual-hop relay link, Figures 2 and 3 plot, respectively, the average SER for 4-PSK and 16QAM versus the average SNR per hop. In both figures, we plot the exact numerical results from simulation using the end-to-end SNR given by (5) for the MRC receiver.

A number of observations on the accuracy of each analytical lower bound of the error performance can be drawn from these graphs. For values of the average SNR per hop less than $10 \mathrm{~dB}$, the geometric mean performance bound gives numerical results that are a little closer to the exact (simulation) results than the minimum SNR bound. However for SNR values equal to or greater than $10 \mathrm{~dB}$, the minimum SNR approximation is more accurate than the geometric mean and its accuracy improves as the average SNR per hop increases. Furthermore, the minimum SNR performance bound is shown to be tight for both values of $N$, although it loses some of its tightness for 


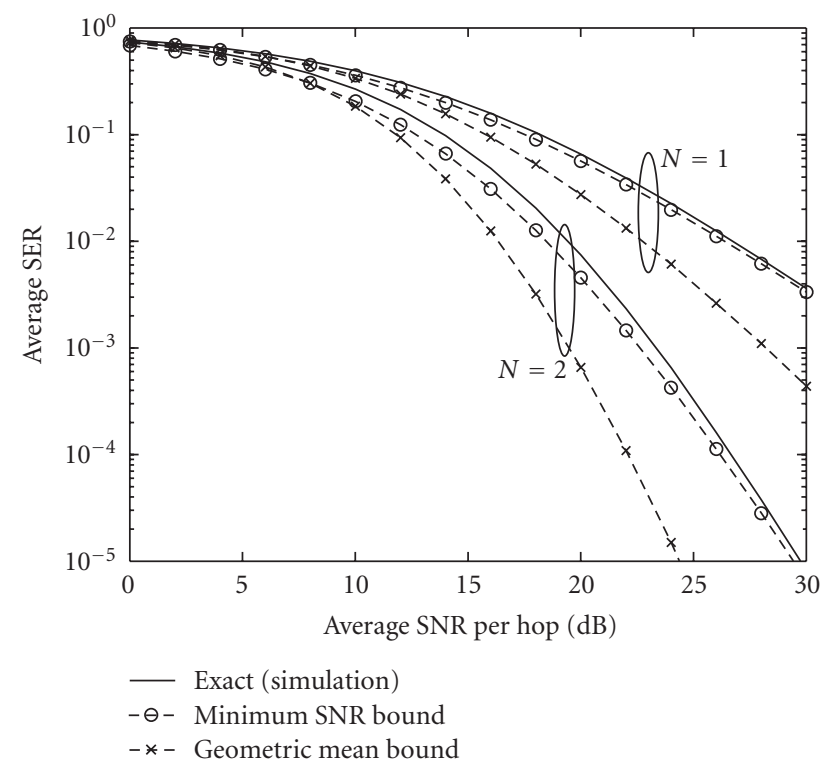

FIGURE 3: Average SER for 16-QAM versus average SNR per hop for $N=1$ and $N=2$ dual-hop links with MRC assuming $m_{0}=1, k_{0}=$ 0.5 for the direct link, $m_{1}=1, k_{1}=1.5$ for the first relay link, and $m_{2}=2, k_{2}=3$ for the second relay link.

$N=2$ compared to $N=1$. However, for both values of $N$, increased values of the average SNR per hop result in the minimum SNR bound to converge to the exact values, whereas the geometric mean bound loses its tightness. Finally, the graphs show the effects of diversity order $N$ and fading parameters $k$ and $m$ on the average SER, that is, the worst performance is obtained for single dual-hop relay and fading conditions with high amount of fading due to shadowing (e.g., $k=1.5$ ) and multipath (e.g., $m=$ $1)$, whereas the SER improves as $N$ increases and fading conditions become less severe (e.g., $k=3$ and $m=2$ for the second relay channel).

\section{Conclusion}

In this paper, we provided closed-form expressions for the MGF of the minimum SNR and geometric mean upper bounds for the end-to-end SNR of a single dual-hop relay system operating over a generalized- $K$ fading environment. Then, for independently faded relay links and MRC diversity receiver, the average SER and BER can be easily evaluated for various modulation schemes using the MGF-based approach. Simulation results of the exact SER were used to verify the analytical results and evaluate the tightness of the two lower performance bounds.

\section{References}

[1] M. O. Hasna and M.-S. Alouini, "End-to-end performance of transmission systems with relays over Rayleigh-fading channels," IEEE Transactions on Wireless Communications, vol. 2, no. 6, pp. 1126-1131, 2003.
[2] M. O. Hasna and M.-S. Alouini, "Harmonic mean and end-toend performance of transmission systems with relays," IEEE Transactions on Communications, vol. 52, no. 1, pp. 130-135, 2004.

[3] T. A. Tsiftsis, G. K. Karagiannidis, S. A. Kotsopoulos, and F.N. Pavlidou, "BER analysis of collaborative dual-hop wireless transmissions," Electronics Letters, vol. 40, no. 11, pp. 679-681, 2004.

[4] S. Ikki and M. H. Ahmed, "Performance analysis of cooperative diversity wireless networks over Nakagami- $m$ fading channel," IEEE Communications Letters, vol. 11, no. 4, pp. 334336, 2007.

[5] S. S. Ikki and M. H. Ahmed, "Performance analysis of dual hop relaying over non-identical weibull fading channelsw," in Proceedings of the 69th IEEE Vehicular Technology Conference (VTC '06), pp. 1-5, Barcelona, Spain, April 2009.

[6] S. Ikki and M. H. Ahmed, "Performance analysis of dualhop relaying communications over generalized gamma fading channels," in Proceedings of IEEE Global Telecommunications Conference (GLOBECOM '07), pp. 3888-3893, Washington, DC, USA, November 2007.

[7] H. A. Suraweera and G. K. Karagiannidis, "Closed-form error analysis of the non-identical Nakagami- $m$ relay fading channel," IEEE Communications Letters, vol. 12, no. 4, pp. 259261, 2008.

[8] H. Suraweera, G. K. Karagiannidis, and P. J. Smith, "Performance analysis of the dual-hop asymmetric fading channel," IEEE Transactions on Wireless Communications, vol. 8, no. 6, pp. 2783-2788, 2009.

[9] P. M. Shankar, "Error rates in generalized shadowed fading channels," Wireless Personal Communications, vol. 28, no. 3, pp. 233-238, 2004.

[10] A. Abdi and M. Kaveh, " $K$ distribution: an appropriate substitute for Rayleigh-lognormal distribution in fadingshadowing wireless channels," Electronics Letters, vol. 34, no. 9, pp. 851-852, 1998.

[11] P. S. Bithas, N. C. Sagias, P. T. Mathiopoulos, G. K. Karagiannidis, and A. A. Rontogiannis, "On the performance analysis of digital communications over Generalized- $K$ fading channels," IEEE Communications Letters, vol. 10, no. 5, pp. 353-355, 2006.

[12] P. S. Bithas, P. T. Mathiopoulos, and S. A. Kotsopoulos, "Diversity reception over generalized- $K\left(K_{G}\right)$ fading channels," IEEE Transactions on Wireless Communications, vol. 6, no. 12, pp. 4238-4243, 2007.

[13] C. K. Datsikas, K. P. Peppas, F. I. Lazarakis, and G. S. Tombras, "Error rate performance analysis of dual-hop relaying transmissions over generalized- $K$ fading channels," AEUInternational Journal of Electronics and Communications. In press.

[14] I. S. Gradshteyn and I. M. Ryzhik, Table of Integrals, Series and Products, Academic Press, New York, NY, USA, 7th edition, 2007.

[15] G. P. Efthymoglou, "On the performance analysis of digital modulations in generalized- $K$ fading channels," submitted to IET Communications, 2010.

[16] “The Wolfram functions site," http://functions.wolfram.com/. 

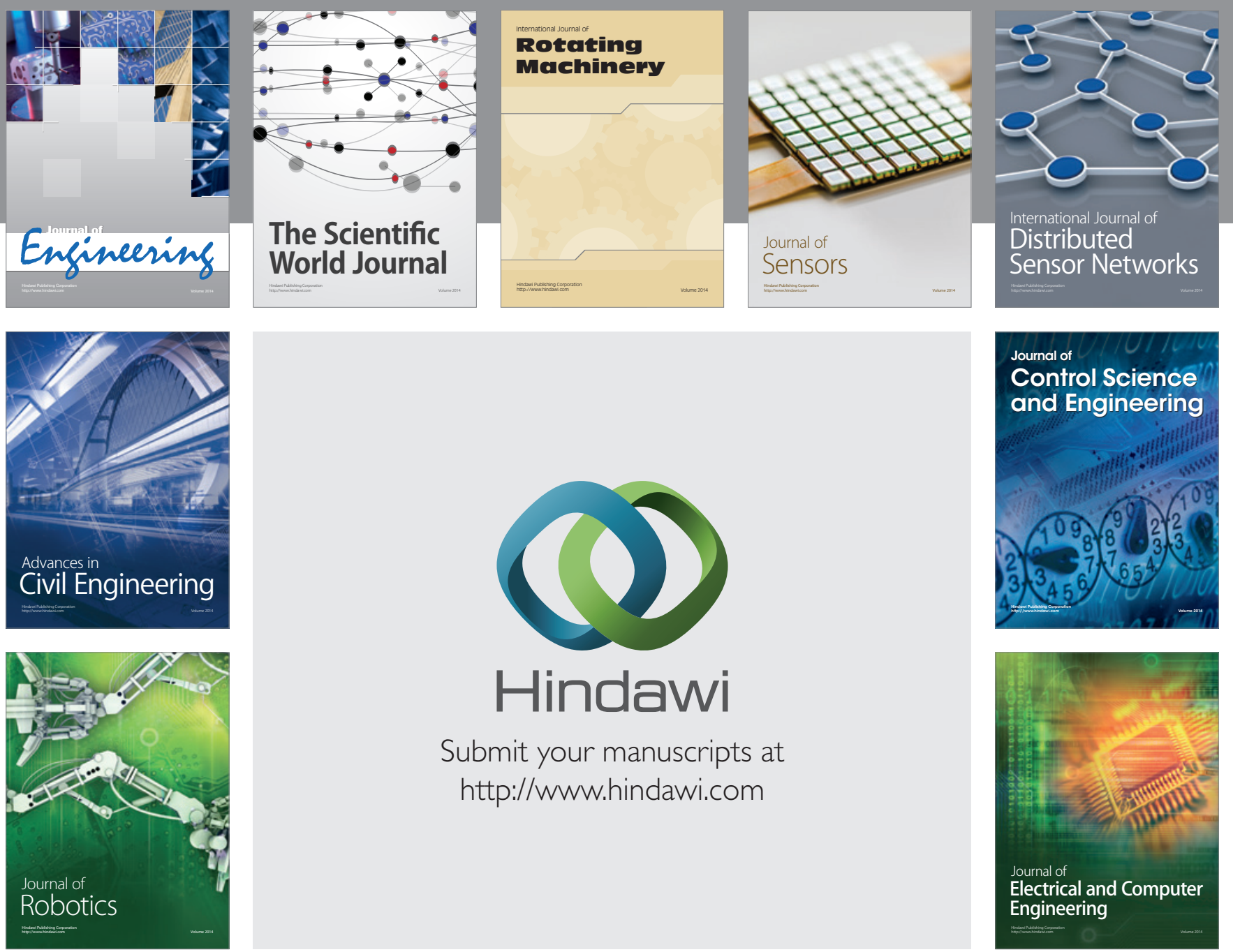

Submit your manuscripts at

http://www.hindawi.com
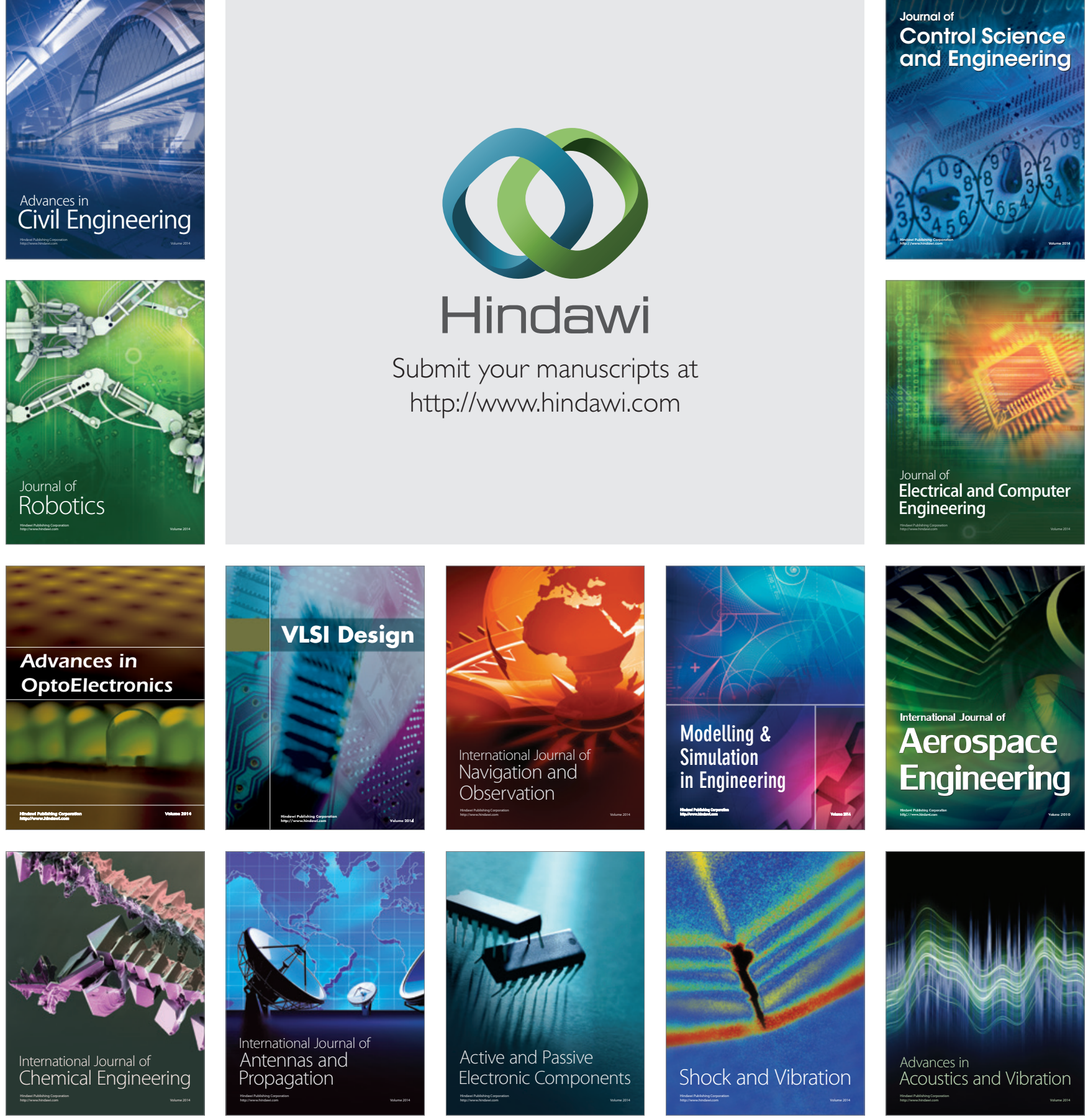